\title{
Patients with adenomyosis are more likely to have deep endometriosis
}

\author{
Midgley Gonzales • Leandro Accardo de Matos • \\ Manoel Orlando da Costa Gonçalves • \\ Roberto Blasbalg • João Antônio Dias Junior • \\ Sérgio Podgaec $\cdot$ Edmund Chada Baracat . \\ Maurício Simões Abrão
}

Received: 14 March 2012 / Accepted: 10 April 2012 /Published online: 3 May 2012

(C) Springer-Verlag 2012

\begin{abstract}
This study seeks to analyze the association between adenomyosis diagnosed by magnetic resonance imaging (MRI) and endometriosis. This is a prospective study of consecutive patients. One hundred fifty-two patients with histologically confirmed endometriosis. Patients were submitted to MRI, then divided into Group A (with adenomyosis) and Group B (without adenomyosis). Thickness of the junctional zone and the presence of intramyometrial cysts were the principal criteria for diagnosis of adenomyosis. MRI data were correlated with clinical status, staging, sites affected, and histological classification of endometriosis. The prevalence of adenomyosis in endometriosis patients was $42.76 \%$. Patients with endometriosis and adenomyosis were more likely than those without adenomyosis to report severe or incapacitating dysmenorrhea $(61.53 \%$ in Group $\mathrm{A} ; 44.83 \%$ in Group $\mathrm{B} ; p=0.041)$ and deep dyspareunia (64.61 \% in Group A; $41.38 \%$ in Group B; $p=0.005$ ), to have stage IV endometriosis (50.77\% in Group A; $33.34 \%$ in Group B; $p=0.03$ ), to have endometriosis of the rectosigmoid (49.23\% in Group A; $32.18 \%$ in Group B; $p=0.033)$,
\end{abstract}

\footnotetext{
M. Gonzales · J. A. Dias Junior · S. Podgaec · E. C. Baracat •

M. S. Abrão $(\bowtie)$

Department of Obstetrics and Gynecology,

University of São Paulo Medical School,

Rua São Sebastião 550,

04708-001 São Paulo, São Paulo, Brazil

e-mail:msabrao@mac.com

L. A. de Matos • M. O. da Costa Gonçalves

Digimagem Diagnósticos,

São Paulo, Brazil

R. Blasbalg

Department of Radiology,

University of São Paulo Medical School,

São Paulo, Brazil
}

and to have undifferentiated or mixed histological types of endometriosis (52.31\% in Group A; $34.48 \%$ in Group B; $p=0.028$ ). A correlation was found between adenomyosis and deep endometriosis with poorer prognosis, particularly endometriosis of the rectosigmoid.

Keywords Endometriosis $\cdot$ Adenomyosis $\cdot$ Magnetic resonance imaging

\section{Introduction}

Adenomyosis is a gynecological disease characterized by the presence of endometrial glands and stroma in the myometrium, generally covered by smooth muscle hyperplasia [1-3]. Its prevalence is unclear, ranging from $15 \%$ to $20 \%$ [4-6] and reaching coefficients of up to $90 \%$ when infertile patients are taken into consideration [7]. Classically, the diagnosis of adenomyosis was based on histology [1, 2]. Morphometric studies show that the myocytes of the subendometrial layer are characterized by a nuclear increase, a reduction in the extracellular matrix and in water volume compared with cells located in the external layer of the myometrium [8]. These cell differences observed in the subendometrial myocytes and around foci of adenomyosis permit identification of the junctional zone and poorly defined low density areas of the myometrium on T2-weighted magnetic resonance imaging (MRI) sequences [8].

The presence of endometrial components in the myometrium may be expressed by an increase in the thickness of the junctional zone on MRI [9]. Most authors agree that a junctional zone thickness $\geq 12 \mathrm{~mm}$ is indicative of a uterus with adenomyosis $[5,10,11]$. The presence of areas of low intensity and poorly defined borders surrounding points of 
high signal intensity on T2-weighted sequences may represent a second criterion for diagnosis [5, 11-15], constituting what are referred to as intramyometrial cysts [5].

Transvaginal ultrasound is the natural first choice of image modality when investigating pelvic pain and endometriosis (including deep lesions) [16, 17]. MRI represents an accurate method for the detection of adenomyosis, sensitivity, and specificity varying from $86 \%$ to $100 \%[5,14$, 18-22], making this a highly effective tool for the diagnosis of this disease [14].

There is a multitude of theories with respect to the association between adenomyosis and endometriosis; however, other investigators consider them to be two distinct entities $[23,24]$. No studies have yet been performed to correlate the two diseases from a diagnostic point of view, taking into consideration the relationship between the ectopic implantation of glands and endometrial stroma in the myometrium, and the sites of implantation and the severity of the foci of endometriosis. Therefore, the objective of the present study was to evaluate the prevalence of adenomyosis in patients with endometriosis and to verify the association of a diagnosis of adenomyosis according to MRI and the following parameters of endometriosis: clinical status, staging [25], the presence of undifferentiation according to histological classification [26] and the sites affected by the disease (peritoneum, ovary, or deep endometriosis).

\section{Methods}

A total of 170 patients consecutively attending the Endometriosis Clinic of the Teaching Hospital of the School of Medicine, University of São Paulo between February 2004 and October 2008 with an indication for videolaparoscopy based on clinical history, physical examination, pelvic echography, and MRI were evaluated according to the protocol used in cases of patients with a clinical suspicion of endometriosis.

In accordance with MRI, the patients were divided into two groups: Group A in which MRI findings were indicative of adenomyosis and Group B in which there were no signs indicative of adenomyosis.

Following surgery, 18 patients were excluded because they did not present endometriosis at laparoscopy and/or histological examination. According to the objectives of our study, to describe the MRI findings in patients with endometriosis, we decided to exclude these patients. The following inclusion criteria were applied to patients in Group A: age 20-45 years, histologically confirmed endometriosis, and MRI findings compatible with a diagnosis of adenomyosis. In addition, the patient should not have been in use of hormones in the 3 months preceding videolaparoscopy (including GnRH analogs, progestogens, and hormonal contraceptives) and should have no contraindications to undergoing MRI. For Group B, the same inclusion criteria were applied as for Group A; however, MRI findings for patients in this group had to indicate an absence of adenomyosis. The study was approved by the institution's Internal Review Board and all the patients read and signed an informed consent form.

With respect to the clinical status of endometriosis, the presence of acyclic pelvic pain, dysmenorrheal, and dyspareunia was recorded, as described by the patients with the use of a visual analog scale. Cyclic bowel and urinary abnormalities (during menstruation) and infertility status were also recorded.

In these patients, endometriosis was classified during laparoscopy as stages I to IV in accordance with the criteria defined by the American Society for Reproductive Medicine (ASRM), 1996 revision [25].

The most important site affected by endometriosis was defined in accordance with the severity of the disease in the affected organ. Three categories were established: peritoneal endometriosis, ovarian endometriosis, and deep endometriosis (lesions with a depth $>5 \mathrm{~mm}$ ), which included retrocervical and bowel lesions as well as lesions of the ureter, bladder, and vagina.

Tissue was analyzed histologically in accordance with the classification patterns proposed by Abrão et al. [26]: stromal, well-differentiated glandular, undifferentiated glandular, or mixed differentiation glandular (presence of epithelia with well-differentiated and undifferentiated patterns at the same site). To facilitate analysis, differential was defined as the presence or absence of endometriosis with an undifferentiated histological pattern, since this is the histological type with the poorest prognosis [26].

All pelvic MRI exams were performed using a 1.5 Tesla GE Signa scanner (General Electric Medical Systems, Milwaukee, WI) or a 1.5 Tesla Philips scanner with an 8channel torso coil or cardiac coil for signal reception and transmission. All the patients had fasted for $4 \mathrm{~h}$ prior to the exam and were placed in the dorsal decubitus position with their arms raised above their heads. Vaginal gel was used when no contraindications were present. Gadolinium contrast medium (10-20 ml) was manually injected intravenously at a speed of approximately $2.0 \mathrm{ml} / \mathrm{s}$.

Morphology of the junctional zone, a subendometrial region of low signal intensity, was evaluated on T1- (axial plane) and T2-weighted sequences (axial, sagittal and coronal planes). T1-weighted sequences were used to detect hemorrhagic foci in the junctional zone and in the myometrium. The other morphological alterations were observed in the T2-weighted sequences.

To determine a diagnosis of adenomyosis by pelvic MRI, the following criteria were taken into consideration $[5,9]$ : focal or diffuse thickening of the junctional zone $\geq 12 \mathrm{~mm}$; the presence of intramyometrial cysts, which consists of a 
high signal area of the myometrium surrounded by an area of low intensity signal with poorly defined borders on T2weighted sequences.

Categorical variables were analyzed using the chi-square test. Fisher's exact test was used whenever the distribution of the variable under analysis rendered the chi-square test inviable. The continuous quantitative variables were compared using Student's $t$ test. All statistical analyses were performed using the SPSS statistical software program (SPSS Inc., USA), adopting a significance level of $5 \%$.

\section{Results}

A total of 152 women with endometriosis (diagnosed by histopathology following videolaparoscopy) were analyzed, $65(42.8 \%)$ of whom were found to have adenomyosis on pelvic MRI (Group A), while 87 (56.2 \%) did not (Group B). The clinical and epidemiological characteristics of the study participants are shown in Table 1 .

An association was found between a diagnosis of adenomyosis (Group A) and a greater likelihood of dysmenorrhea (severe or incapacitating) and deep dyspareunia. No differences were found between the two groups with respect to any of the other symptoms and there were no differences in findings detected at physical examination.

Table 1 Epidemiological and clinical characteristics of the patients in Group A (with adenomyosis) compared to the patients in Group B (without adenomyosis)

\begin{tabular}{|c|c|c|c|}
\hline & $\begin{array}{l}\text { Group A } \\
(N=65)\end{array}$ & $\begin{array}{l}\text { Group B } \\
(N=87)\end{array}$ & $p$ value \\
\hline \multicolumn{4}{|l|}{ Skin color } \\
\hline Yellow & $4(6.15 \%)$ & $8(9.19 \%)$ & \\
\hline White & $47(72.30 \%)$ & $58(66.67 \%)$ & \\
\hline Black & $14(21.55 \%)$ & $21(24.14 \%)$ & 0.699 \\
\hline \multicolumn{4}{|l|}{ Pregnancies } \\
\hline 0 & $41(63.10 \%)$ & $56(64.37 \%)$ & \\
\hline 1 & $20(30.77 \%)$ & $26(29.88 \%)$ & \\
\hline 2 & $4(6.15 \%)$ & $4(4.60 \%)$ & 0.717 \\
\hline Mean age & 33.43 & 32.82 & 0.474 \\
\hline \multicolumn{4}{|l|}{ Clinical data } \\
\hline Dysmenorrhea & $40(61.53 \%)$ & $39(44.83 \%)$ & $0.041^{*}$ \\
\hline $\begin{array}{l}\text { Acyclic pelvic } \\
\text { pain }\end{array}$ & $22(33.85 \%)$ & $19(21.84 \%)$ & 0.099 \\
\hline Dyspareunia & $42(64.61 \%)$ & $36(41.38 \%)$ & 0.005 \\
\hline Infertility & $31(47.69 \%)$ & $41(47.13 \%)$ & 0.945 \\
\hline $\begin{array}{l}\text { Cyclic bowel } \\
\text { symptoms }\end{array}$ & $32(49.23 \%)$ & $32(36.78 \%)$ & 0.124 \\
\hline $\begin{array}{l}\text { Cyclic urinary } \\
\text { symptoms }\end{array}$ & $3(4.61 \%)$ & $7(8.04 \%)$ & 0.517 \\
\hline
\end{tabular}

$* p<0.05$
When the staging of endometriosis obtained during videolaparoscopy in the 152 patients was compared between the two study groups, a relationship was found between adenomyosis and advanced stages of endometriosis (Table 2). This association was present when patients with stages III and IV were grouped together and became even more expressive when the presence of adenomyosis was investigated only in patients with stage IV endometriosis. Table 3 shows the distribution of the patients with and without adenomyosis according to the site most severely affected by pelvic endometriosis.

In the evaluation of the histological type of endometriosis (Table 4), the presence of undifferentiated or mixed histological patterns of endometriosis in the tissue studied was significantly more common in the patients with adenomyosis (Group A; $p=0.028$ ).

\section{Discussion}

Great advances have been made in the field of diagnosis and in the surgical treatment of deep endometriosis. In addition, new aspects are now beginning to be understood within the realm of etiopathogeny. Little is known about adenomyosis. There is an intuitive association with endometriosis. The tissues involved, the sites affected, the clinical status all connect these two diseases; however, a more objective confirmation of this association is required. Evaluating the characteristics of the two study groups, no differences were found between the two with respect to epidemiological parameters. There was a predominance of white patients, which is in agreement with data published by Arruda et al. [27]. The homogeneity found in the present study reinforces the power of this present analysis, since the only difference between the two groups was the variable under investigation: a diagnosis of adenomyosis on MRI.

The purpose of using imaging exams to diagnose adenomyosis is to study the disease in patients in whom hysterectomy is not indicated. This became feasible and MRI is

Table 2 Evaluation of the patients in Group A (with adenomyosis) compared with the patients in Group B (without adenomyosis), according to the stage of endometriosis (ASRM, 1996)

\begin{tabular}{lrcl}
\hline Stage & Group A $(N=65)$ & Group B $(N=87)$ & $p$ value \\
\hline I & $9(13.85 \%)$ & $26(29.87 \%)$ & $0.020^{*}$ \\
II & $14(21.54 \%)$ & $19(21.84 \%)$ & 0.965 \\
I/II & $23(35.38 \%)$ & $45(51.72 \%)$ & $0.045^{*}$ \\
III & $9(13.85 \%)$ & $13(14.49 \%)$ & 0.849 \\
IV & $33(50.77 \%)$ & $29(33.34 \%)$ & $0.030^{*}$ \\
III/IV & $42(64.61 \%)$ & $42(48.27 \%)$ & $0.045^{*}$ \\
\hline
\end{tabular}

${ }^{*} p<0.05$ 
Table 3 Evaluation of the patients in Group A (with adenomyosis) in relation to the patients in Group B (without adenomyosis), according to the site of the endometriotic lesion

\begin{tabular}{lccc}
\hline Site & Group A $(N=65)$ & Group B $(N=87)$ & $p$ value \\
\hline Peritoneal & $26(40 \%)$ & $49(56.32 \%)$ & $0.046^{*}$ \\
Ovarian & $42(64.61 \%)$ & $45(51.72 \%)$ & 0.112 \\
Deep & & & \\
Retrocervical & $39(60 \%)$ & $34(39.08 \%)$ & $0.011^{*}$ \\
Vagina & $5(7.69 \%)$ & $4(4.60 \%)$ & 0.498 \\
Bladder & $6(9.23 \%)$ & $8(9.19 \%)$ & 0.994 \\
Ureter & $6(9.23 \%)$ & $3(3.45 \%)$ & 0.172 \\
Rectosigmoid & $32(49.23 \%)$ & $28(32.18 \%)$ & $0.033^{*}$ \\
\hline
\end{tabular}

${ }^{*} p<0.05$

currently considered to be a highly accurate tool for the diagnosis of this pathology $[7,11,12,14,28]$. The radiological criteria selected in the present study for the diagnosis of adenomyosis were those most generally accepted in the literature: thickness of the junctional zone $\geq 12 \mathrm{~mm}$ and the presence of intramyometrial cysts, regions of hyperintense signals surrounded by an area of low intensity signals and poorly defined borders on T2-weighted sequences [5, 14, 19-21]. In the 152 patients with a diagnosis of endometriosis in the present study, critical analysis of their MRI scans revealed that 65 patients $(42.8 \%)$ had a diagnosis of adenomyosis. The coefficient depends to a great extent on the characteristics, principally the clinical characteristics of the study population $[1,29]$.

The prevalence of adenomyosis in patients with endometriosis has been evaluated in few studies. Bazot et al. [30] reported a prevalence of adenomyosis of $27 \%$ in patients with endometriosis. On the other hand, Kunz et al. [7] reported a prevalence of $79 \%$, which increased to $90 \%$ when adenomyosis was diagnosed in patients with endometriosis and female infertility. Moreover, Larsen et al. [31] found that in $34.6 \%$ of patients with endometriosis had adenomyosis compared to $19.4 \%$ in the control patients. In a recent study published by Dai et al. [32], adenomyosis

Table 4 Evaluation of the patients in Group A (with adenomyosis) in relation to the patients in Group B (without adenomyosis), according to the presence of undifferentiated histological pattern in the endometriotic lesion

\begin{tabular}{lll}
\hline Histology & $\begin{array}{l}\text { Group A } \\
(N=65)\end{array}$ & $\begin{array}{l}\text { Group B } \\
(N=87)\end{array}$ \\
\hline $\begin{array}{l}\text { Well-differentiated glandular or } \\
\text { stromal disease }\end{array}$ & $31(47.69 \%)$ & $57(65.52 \%)$ \\
$\begin{array}{l}\text { Undifferentiated glandular or mixed } \\
\text { disease }\end{array}$ & $34(52.31 \%)$ & $30(34.48 \%)$ \\
\hline
\end{tabular}

$p=0.028$ was diagnosed in $15.8 \%$ of patients presenting deep endometriosis and it was significantly higher than in patients without deep endometriosis.

Parker et al. [28] evaluated chronic pelvic pain in women with endometriosis and reported a prevalence of adenomyosis, as diagnosed by MRI, of $40 \%$. In the present study, the patients were symptomatic with respect to endometriosis, meeting the criteria for an indication for videolaparoscopy. Therefore, the $42.8 \%$ prevalence of adenomyosis in endometriosis patients that was found in this study would appear to be compatible with the data in the literature considering the characteristics of the study population. Furthermore, there were statistically more cases of severe or incapacitating dysmenorrhea and deep dyspareunia in the group of patients with adenomyosis compared to the group with a diagnosis of endometriosis alone. It is comprehensible that dysmenorrhea would be more severe in this group bearing in mind the factors triggered by the combination of these two pathologies. Dyspareunia was more intense in patients of Group A, probably as a result of the associated deep endometriotic lesions, since the severity of symptoms is directly associated with the depth of the lesions [33-35]. Therefore, as has been shown in cases of endometriosis in which there is a significant correlation between deep dyspareunia and retrocervical endometriotic lesions [36], the severity of the symptoms in adenomyosis also correlates with the extension and depth of the myometrial invasion of the lesion [37, 38].

With respect to the endometriosis staging classification system proposed by the ASRM in 1985 and revised in 1996 [25], adenomyosis was found to be associated with more advanced stages of endometriosis, particularly stage IV of the disease. These data are in agreement with a study published by Kunz et al. [7] in which these authors correlated moderate and severe endometriosis with a junctional zone thickness of $12.4 \mathrm{~mm}$ (adenomyosis), with $p=0.02$. The stage IV was also found among women with severe endometriosis, $42.8 \%$ had adenomyosis, with deeper wall invasion, compared to $29.4 \%$ in the women with other stages of endometriosis [31].

Considering the site affected by endometriosis and its association with adenomyosis, a greater association was found between adenomyosis and cases of deep retrocervical endometriosis $(60 \% ; p=0.01)$ and those in which endometriosis was affecting the rectosigmoid $(49.2 \% ; p=0.03)$. Parker et al. [28] also suggested an association between adenomyosis and deep endometriosis; however, these authors emphasized that due to the small sample size in their study, it was impossible to correlate the data with the stage of endometriosis.

With respect to the histological classification of the disease [26], there was a significantly greater incidence of undifferentiated endometriosis, either in the pure or mixed form, in the group of patients with adenomyosis compared 
to the group of patients with endometriosis alone ( $p=0.028)$. These data are compatible with the finding that patients with undifferentiated endometriosis have more severe forms of endometriosis $[26,39]$.

These findings confirm previous data correlating adenomyosis with deep endometriosis (principally bowel endometriosis) and with advanced stages of the disease [25]. An association was therefore established between adenomyosis and cases of endometriosis with poor prognosis.

Despite the lack of studies in which patients with endometriosis were analyzed according to the present format, particularly correlating endometriosis with adenomyosis, it is worth mentioning the publication of Landi et al. [11], who reported poorer prognosis in patients with concurrent adenomyosis and endometriosis. However, they did not establish any satisfactory association with staging or the site of foci of endometriosis. Peritoneal lesions present as epithelial glands covered with endometrial-type stroma. This feature is absent in deep lesions that involve smooth muscle tissue [40, 41] similar to adenomyosis lesions. In the present study, an association was found between adenomyosis and deep endometriosis. It should be emphasized that it is the deep rather than the superficial endometriotic foci that resemble those found in adenomyosis [42].

When the three different forms of endometriosis, peritoneal, ovarian, and that affecting the rectovaginal septum were defined, Nisolle and Donnez [43] attributed different etiopathogenies to the peritoneal form of the disease (implants resulting from retrograde menstruation) and to the deep lesions, said to correspond to an adenomyotic nodule originating from Müllerian remnants by means of metaplasia $[40,41,44]$. These resemble an adenomyoma, an accumulation of smooth muscle tissue and endometrial glands with scarce stroma [40, 41, 44]. Well-grounded studies have shown that the nodules of deep retrocervical, and bowel endometriosis are not the result of deep infiltrative endometriosis but are similar to the nodules of adenomyosis that develop from Müllerian remnants by metaplasia [40, 41, 44].

As deep endometriosis, adenomyosis has also been explained $[4,45]$ as a condition originating from Müllerian remnants by metaplasia. These authors presume that there are transitional quiescent Müllerian elements between the endometrium and the myometrium that would have the capacity to develop by metaplasia.

The close association found in this study between adenomyosis and deep endometriosis, associated with advanced stages and pure or mixed undifferentiated histological patterns, permits some common theories to be expounded on the genesis of endometriosis and that of adenomyosis. According to the present findings, adenomyosis may be interpreted as a marker for deep endometriosis. Nevertheless, more studies need to be performed to supply further useful information and a better understanding of the association between these two diseases, providing greater knowledge on their etiopathogenies and developing new instruments for clinical practice.

Declaration of interest The authors report no conflicts of interest. The authors alone are responsible for the content and writing of the paper.

\section{References}

1. Bird CC, McElin TW, Manalo-Estrella P (1972) The elusive adenomyosis of the uterus-revisited. Am J Obstet Gynecol 112:583-593

2. Ferenczy A (1998) Pathophysiology of adenomyosis. Hum Reprod Update 4:312-322

3. Leyendecker G, Kunz G, Noe M et al (1998) Endometriosis: a dysfunction and disease of the archimetra. Hum Reprod Update 4:752-762

4. Racinet C, Morin X, Martin H (1987) Adenomyosis. Contrib Gynecol Obstet 16:183-191

5. Reinhold C, McCarthy S, Bret PM et al (1996) Diffuse adenomyosis: comparison of endovaginal US and MR imaging with histopathologic correlation. Radiology 199:151-158

6. Dueholm M, Lundorf E (2007) Transvaginal ultrasound or MRI for diagnosis of adenomyosis. Curr Opin Obstet Gynecol 19:505-512

7. Kunz G, Beil D, Huppert P et al (2005) Adenomyosis in endometriosis - prevalence and impact on fertility. Evidence from magnetic resonance imaging. Hum Reprod 20:2309-2316

8. Fusi L, Cloke B, Brosens JJ (2006) The uterine junctional zone. Best Pract Res Clin Obstet Gynaecol 20:479-491

9. Hricak H, Alpers C, Crooks LE et al (1983) Magnetic resonance imaging of the female pelvis: initial experience. AJR Am J Roentgenol 141:1119-1128

10. Bazot M, Cortez A, Darai E et al (2001) Ultrasonography compared with magnetic resonance imaging for the diagnosis of adenomyosis: correlation with histopathology. Hum Reprod 16:2427-2433

11. Landi S, Mereu L, Pontrelli G et al (2008) The influence of adenomyosis in patients laparoscopically treated for deep endometriosis. J Minim Invasive Gynecol 15:566-570

12. Reinhold C, Tafazoli F, Wang L (1998) Imaging features of adenomyosis. Hum Reprod Update 4:337-349

13. Stratton P, Winkel CA, Sinaii N et al (2002) Location, color, size, depth, and volume may predict endometriosis in lesions resected at surgery. Fertil Steril 78:743-749

14. Ascher SM, Arnold LL, Patt RH et al (1994) Adenomyosis: prospective comparison of MR imaging and transvaginal sonography. Radiology 190:803-806

15. Tamai K, Togashi K, Ito T, Morisawa N et al (2005) MR imaging findings of adenomyosis: correlation with histopathologic features and diagnostic pitfalls. Radiographics 25:21-40

16. Gonçalves MO, Podgaec S, Dias JA Jr et al (2010) Transvaginal ultrasonography with bowel preparation is able to predict the number of lesions and rectosigmoid layers affected in cases of deep endometriosis, defining surgical strategy. Hum Reprod 25:665-671

17. Gonçalves MO, Dias JA Jr, Podgaec S et al (2009) Transvaginal ultrasound for diagnosis of deeply infiltrating endometriosis. Int $\mathrm{J}$ Gynaecol Obstet 104:156-160

18. Mark AS, Hricak H, Heinrichs LW et al (1987) Adenomyosis and leiomyoma: differential diagnosis with MR imaging. Radiology 163:527-529 
19. Togashi K, Nishimura K, Itoh K et al (1988) Adenomyosis: diagnosis with MR imaging. Radiology 166:111-114

20. Togashi K, Ozasa H, Konishi I et al (1989) Enlarged uterus: differentiation between adenomyosis and leiomyoma with MR imaging. Radiology 171:531-534

21. Hricak H, Finck S, Honda G et al (1992) MR imaging in the evaluation of benign uterine masses: value of gadopentetate dimeglumine-enhanced T1-weighted images. AJR Am J Roentgenol 158:1043-1050

22. Chamie LP, Blasbalg R, Gonçalves MO et al (2009) Accuracy of magnetic resonance imaging for diagnosis and preoperative assessment of deeply infiltrating andometriosis. Int J Gynaecol Obstet 106:198-201

23. Ridley JH (1968) The histogenesis of endometriosis. A review of facts and fancies. Obstet Gynecol Surv 23:1-35

24. Greaves P, White IN (2006) Experimental adenomyosis. Best Pract Res Clin Obstet Gynaecol 20:503-510

25. American Society for Reproductive Medicine (1997) Revised American Society for Reproductive Medicine classification of endometriosis: 1996. Fertil Steril 67:817-821

26. Abrão MS, Neme RM, Carvalho FM et al (2003) Histological classification of endometriosis as a predictor of response to treatment. Int J Gynaecol Obstet 82:31-40

27. Arruda MS, Petta CA, Abrão MS et al (2003) Time elapsed from onset of symptoms to diagnosis of endometriosis in a cohort study of Brazilian women. Hum Reprod 18:756-759

28. Parker JD, Leondires M, Sinaii N et al (2006) Persistence of dysmenorrhea and nonmenstrual pain after optimal endometriosis surgery may indicate adenomyosis. Fertil Steril 86:711-715

29. Atri M, Reinhold C, Mehio AR et al (2000) Adenomyosis: US features with histologic correlation in an in-vitro study. Radiology 215:783-790

30. Bazot M, Fiori O, Darai E (2006) Adenomyosis in endometriosisprevalence and impact on fertility. Evidence from magnetic resonance imaging. Hum Reprod 21:1101-1102

31. Larsen SB, Lundorf E, Forman A et al (2011) Adenomyosis and junctional zone changes in patients with endometriosis. Eur $\mathrm{J}$ Obstet Gynecol Reprod Biol 157:206-211

32. Dai Y, Leng JH, Lang JH et al (2012) Anatomical distribution of pelvic deep infiltrating endometriosis and its relationship with pain symptoms. Chin Med J (Engl) 125:209-213
33. Koninckx PR, Meuleman C, Demeyere S et al (1991) Suggestive evidence that pelvic endometriosis is a progressive disease, whereas deeply infiltrating endometriosis is associated with pelvic pain. Fertil Steril 55:759-765

34. Porpora MG, Koninckx PR, Piazze J et al (1999) Correlation between endometriosis and pelvic pain. J Am Assoc Gynecol Laparosc 6:429-434

35. Chapron C, Fauconnier A, Dubuisson JB et al (2003) Deep infiltrating endometriosis: relation between severity of dysmenorrhea and extent of disease. Hum Reprod 18:760-766

36. Berbel BT, Podgaec S, Abrão MS (2008) Analysis of the association between symptoms referred by patients with endometriosis and the site of the disease. Rev Med (São Paulo) 87:195-200

37. Benson RC, Sneeden VD (1958) Adenomyosis: a reappraisal of symptomatology. Am J Obstet Gynecol 76:1044-1061

38. Nishida M (1991) Relationship between the onset of dysmenorrhea and histologic findings in adenomyosis. Am J Obstet Gynecol 165:229-231

39. Kamergorodsky G, Ribeiro PAA, Galvão MAL et al (2007) Avaliação da classificação histológica da endometriose observada em implantes de mulheres portadoras de endometriose pélvica superficial e profunda. RBGO 29:568-574

40. Nisolle M (1996) Peritoneal, ovarian and rectovaginal endometriosis are three distinct entities. Dissertation. Catholic University of Louvain

41. Nisolle M, Donnez J (1996) Peritoneal, ovarian and rectovaginal endometriosis: the identification of three separate diseases. Carnforth: Parthenon Publishing

42. Somigliana E, Infantino M, Candiani M et al (2004) Association rate between deep peritoneal endometriosis and other forms of the disease: pathogenetic implications. Hum Reprod 19:168-171

43. Nisolle M, Donnez J (1997) Peritoneal endometriosis, ovarian endometriosis, and adenomyotic nodules of the rectovaginal septum are three different entities. Fertil Steril 68:585-596

44. Donnez J, Nisolle M, Casanas-Roux F et al (1996) Stereometric evaluation of peritoneal endometriosis and endometriotic nodules of the rectovaginal septum. Hum Reprod 11:224228

45. Minh HN, Smadja A, Orcel L (1986) An integrated histogenetic concept of internal and external endometriosis. J Gynecol Obstet Biol Reprod 15:29-35 